\title{
Presentaciones atípicas de histoplasmosis
}

\section{Atypical presentations of histoplasmosis}

\author{
María Eugenia Landaeta, Maria Teresa Colella, Jocays Caldera, \\ Arantza Roselló, Sofía Mata-Essayag • Caracas (Venezuela)
}

\section{Resumen}

La histoplasmosis es una enfermedad granulomatosa, producida por Histoplasma capsulatum. Causa un amplio espectro de manifestaciones clínicas, la más frecuente es la pulmonar. En algunos casos se presentan formas diseminadas con afectación de uno o múltiples órganos, de características atípicas difíciles de reconocer. La patogenia de esta enfermedad es igual a la de tuberculosis y se ha descrito coinfección. Se reportan tres casos de histoplasmosis de presentación infrecuente: ancianos, coinfección con micobacterias, localizaciones atípicas y revisión de la literatura. El primer caso fue el de un paciente de 19 años de edad con absceso cerebral. El segundo y tercer caso correspondieron a personas mayores de 60 años, con coinfección por Histoplasma capsulatum y Mycobacterium sp, de presentación articular y enfermedad ginecológica, respectivamente. En Venezuela, zona endémica para esta micosis, el médico está obligado a tener un alto índice de sospecha acerca de la misma, teniendo en cuenta factores como la edad y la epidemiología. (Acta Med Colomb 2015; 40: 254-259).

Palabras clave: histoplasmosis, tuberculosis, absceso cerebral, artritis séptica, Mycobacterium tuberculosis, itraconazol.

\begin{abstract}
Histoplasmosis is a granulomatous disease caused by Histoplasma capsulatum. It causes a wide spectrum of clinical manifestations, being the pulmonary the most common one. In some cases disseminated forms with involvement of one or multiple organs are manifested with atypical characteristics difficult to recognize. The pathogenesis of this disease is equal to that of TB and co-infection has been described. Three cases of infrequent presentation of histoplasmosis: elderly, co-infection with mycobacteria, atypical presentations and review of the literature are presented. The first case involved a 19-year-old with cerebral abscess. The second and third cases were for people over 60, co-infected with Histoplasma capsulatum and Mycobacterium sp, with joint involvement and gynecological disease, respectively. In Venezuela, endemic area for histoplasmosis, the doctor is required to have a high index of suspicion about this entity, taking into account factors such as age and epidemiology. (Acta Med Colomb 2015; 40: 254-259).
\end{abstract}

Keywords: histoplasmosis, tuberculosis, brain abscess, septic arthritis, Mycobacterium tuberculosis, itraconazole.
Dra. María Eugenia Landaeta: MSc. Sección de Micología Médica "Dr. Dante Borelli", Instituto de Medicina Tropical, Universidad Central de Venezuela; Dra. Maria Teresa Colella: BSc. Sección de Micología Médica "Dr. Dante Borelli”, Instituto de Medicina Tropical, Universidad Central de Venezuela; Dra. Jocays Caldera: Servicio de Infectología, Hospital Universitario de Caracas, Venezuela; Dra. Arantza Roselló: BSc. Sección de Micología Médica "Dr. Dante Borelli", Instituto de Medicina Tropical, Universidad Central de Venezuela; Dra. Sofía Mata-Essayag: MSc PhD. Sección de Micología Médica “Dr. Dante Borelli”, Instituto de Medicina Tropical, Universidad Central de Venezuela. Caracas (Venezuela).

Correspondencia. Dra. María Eugenia Landaeta. Caracas (Venezuela)

E-mail: maria.landaeta@ucv.ve

Recibido: 26/IX/14 Aceptado: 20/IX/15

\section{Introducción}

La histoplasmosis es una enfermedad granulomatosa, producida por Histoplasma capsulatum y tiene una distribución cosmopolita (1).

El espectro de presentaciones de la enfermedad es una consecuencia de las relaciones entre diversos factores como el tamaño del inóculo, el grado de exposición a las conidias del hongo y las condiciones del huésped. La puerta de entrada en la infección natural es el tracto respiratorio, desde donde se inicia una infección primaria, por lo general autolimitada. El individuo infectado porta los microorganismos durante muchos años después de esta primoinfección, sin presentar signos ni síntomas de enfermedad. Esta es usualmente subclínica excepto en pacientes inmunocomprometidos, como los enfermos de SIDA, aquellos con enfermedades hematooncológicas, trasplantados, desnutridos, lactantes y ancianos (1).

Histoplasma capsulatum causa un amplio espectro de manifestaciones clínicas, desde una enfermedad catarral, hasta la enfermedad pulmonar cavitaria crónica y en algunos casos formas diseminadas, que pueden tener afectación de uno o múltiples órganos, presentándose de forma atípica 
difícil de reconocer. Aunque estas últimas pueden comprometer la vida del paciente, la mayoría de los individuos se recuperan sin presentar complicaciones cuando son tratados oportunamente (1).

La histoplasmosis tiene la misma patogenia de otras enfermedades granulomatosas crónicas, como es el caso de la tuberculosis y otras micosis. Por este motivo no es de extrañar que en algunas ocasiones se observe la coexistencia de ambos microorganismos, principalmente en pacientes inmunocomprometidos, aunque también puede ocurrir en personas inmunocompetentes (2).

En este reporte se presentan tres casos de esta entidad de características infrecuentes: ancianos, coinfección con Mycobacterium spp y localizaciones atípicas.

\section{Caso 1}

\section{Casos clínicos}

Masculino de 19 años de edad, consulta por presentar tos, fiebre, expectoración y disnea leve. Radiografía: infiltrado trabéculonodular bilateral. Recibió antibióticos sin mejoría, persistencia de fiebre, anorexia, pérdida de peso, cefalea, somnolencia y convulsiones, en tres meses. Tomografía axial computarizada: absceso cerebral en región occipital superior derecha. Laboratorio: anemia, leucopenia y elevación de reactantes de fase aguda, ELISA para VIH negativo. Fue llevado a craneotomía y drenaje de absceso, con toma de muestra de secreción purulenta para estudio micológico. Coloración de Giemsa: levaduras intracelulares sugestivas de Histoplasma capsulatum. Inmunodifusión doble: positiva (Figura 1). En vista de estos hallazgos, se reinterroga al paciente, quien entonces informó sobre visita a cueva en Boconó, Estado Trujillo, área endémica para histoplasmosis en Venezuela, 15 días antes del inicio de los síntomas. Se indicó anfotericina B deoxicolato, seguido por itraconazol con mejoría. Se repite la inmunodifusión doble para seguimiento después de cinco meses, la cual resulta negativa. Se continuó tratamiento por un año encontrándose asintomático dos años después.

\section{Caso 2}

Masculino de 61 años, quien un año y medio antes del ingreso, presentó aumento de volumen y dolor en rodilla derecha, seis meses después tumoración en la región poplítea, con dolor y limitación funcional; se realizó artrocentesis con mejoría parcial. Al examen físico se encontró quiste de Baker e inflamación de rodilla derecha; limitación para la flexoextensión. El gammagrama óseo de tres fases evidenció hipercaptación en la interlínea articular. La resonancia magnética nuclear (RMN) de rodilla mostró lesión de ocupación de espacio (LOE) quístico en región poplítea, quiste de Baker $14 \mathrm{~cm}$, severos cambios osteodegenerativos gonartrósicos, signos de osteonecrosis, meniscopatía

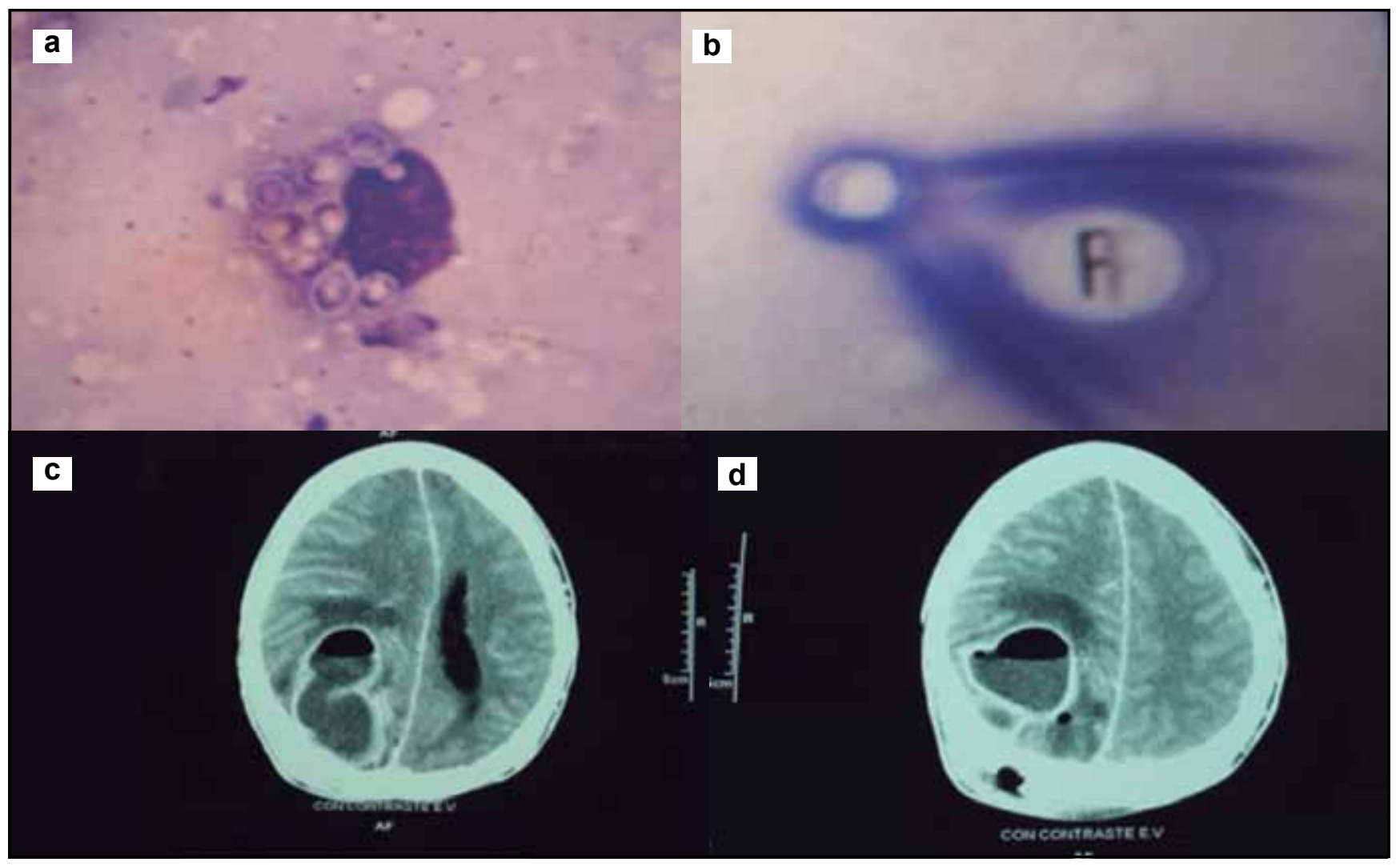

Figura 1. Absceso cerebral. a) Levaduras intracelulares compatibles con Histoplasma capsulatum-coloración Giemsa 100x. b) Inmunodifusión doble - Bandas H y M. c) y d) Tomografía Computarizada - absceso cerebral localizado en la región occipital derecha. 
degenerativa (Figura 2). Laboratorio: neutrofilia, anemia normocítica normocrómica, aumento de velocidad de sedimentación globular y proteína $\mathrm{C}$ reactiva. ELISA para VIH negativo. Se realizó limpieza quirúrgica de la articulación con toma de muestras. Líquido articular: pleocitosis con predominio de células mononucleares. Ziehl Neelsen: se observaron bacilos ácidorresistentes. Cultivo: Mycobacterium tuberculosis. Giemsa: levaduras intracelulares sugestivas de Histoplasma sp. Cultivo: H. capsulatum. Serología (inmunodifusión doble): negativa. Tratamiento: anfotericina B deoxicolato hasta el diagnóstico de tuberculoso. Se omitió el antifúngico y se inició tratamiento antituberculoso con cuatro drogas (isoniacida, etambutol, pirazinamida, rifampicina) por dos meses, seguido de rifampicina e isoniacida por cuatro meses. La evolución clínica fue tórpida con recaída de artromielitis, posiblemente explicado por el abandono del tratamiento antifúngico. Se realizó resección ósea de cóndilos femorales y meseta tibial, iniciando itraconazol, con mejoría de síntomas y de parámetros de laboratorio.

\section{Caso 3}

Femenina de 74 años, consultó por presentar historia de seis meses con pérdida de peso de $10 \mathrm{~kg}$ y aumento de volumen abdominal. Antecedentes personales: hipertensión arterial, hábito tabáquico desde temprana edad, depresión reactiva. Además refirió limpieza reciente de local con abundantes excretas de palomas. Vivió en una granja avícola (de gallinas ponedoras) en la infancia. Tomografía axial computarizada abdominopélvica: abundante líquido libre en cavidad. Radiografía de tórax: neumonitis intersticial y nódulo pulmonar a nivel del quinto arco intercostal anterior izquierdo. Prueba de tuberculina: $25 \mathrm{~mm}$. Hemoglobina

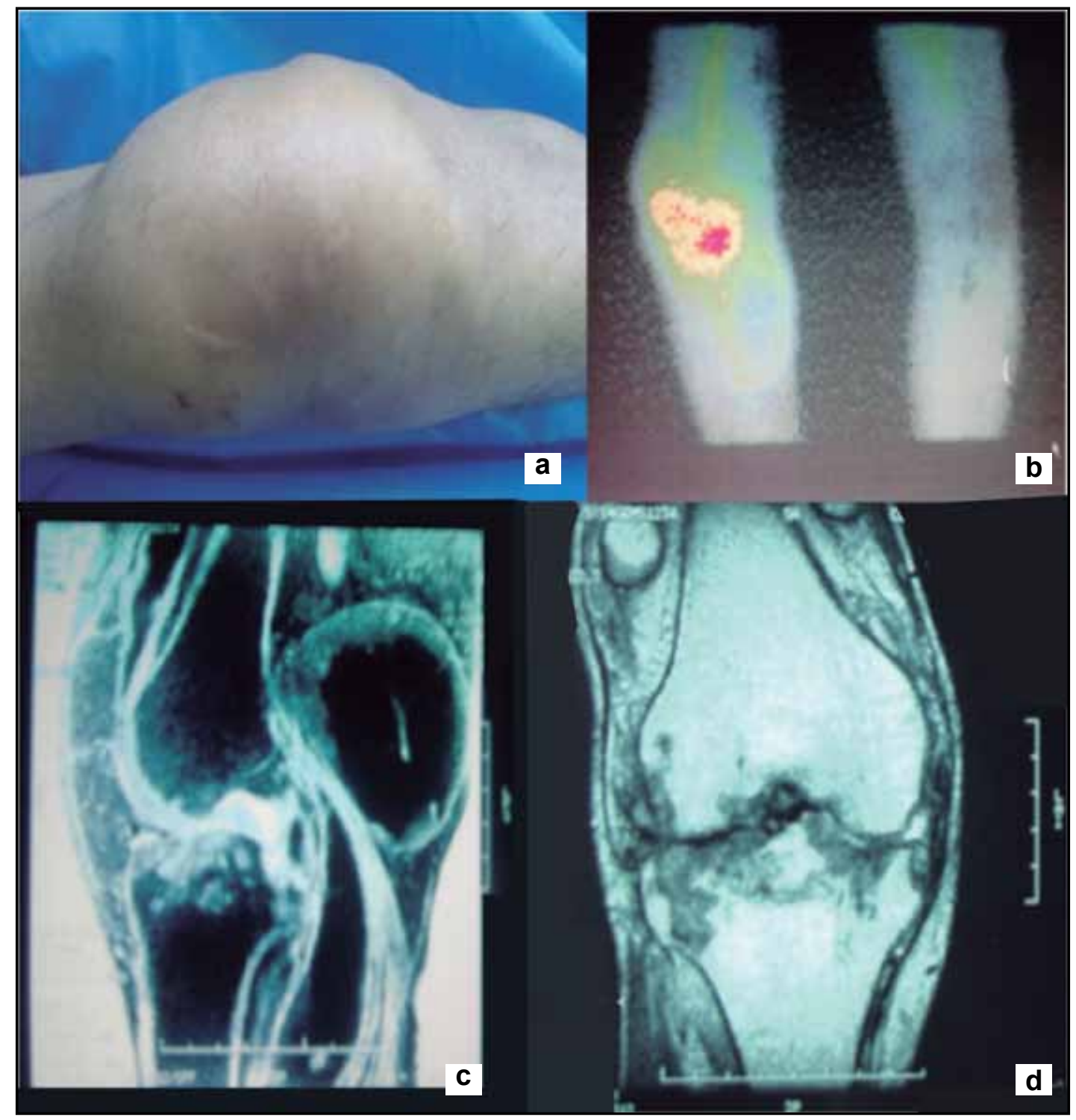

Figura 2. Artritis de rodilla. a) Aspecto clínico. b) Gammagrama óseo. c) RMN rodilla derecha: LOE quístico en región poplítea, Quiste de Baker 14 cm. d) RMN rodilla derecha: Severos cambios osteodegenerativos gonartrósicos, signos de osteonecrosis. Meniscopatía degenerativa. 


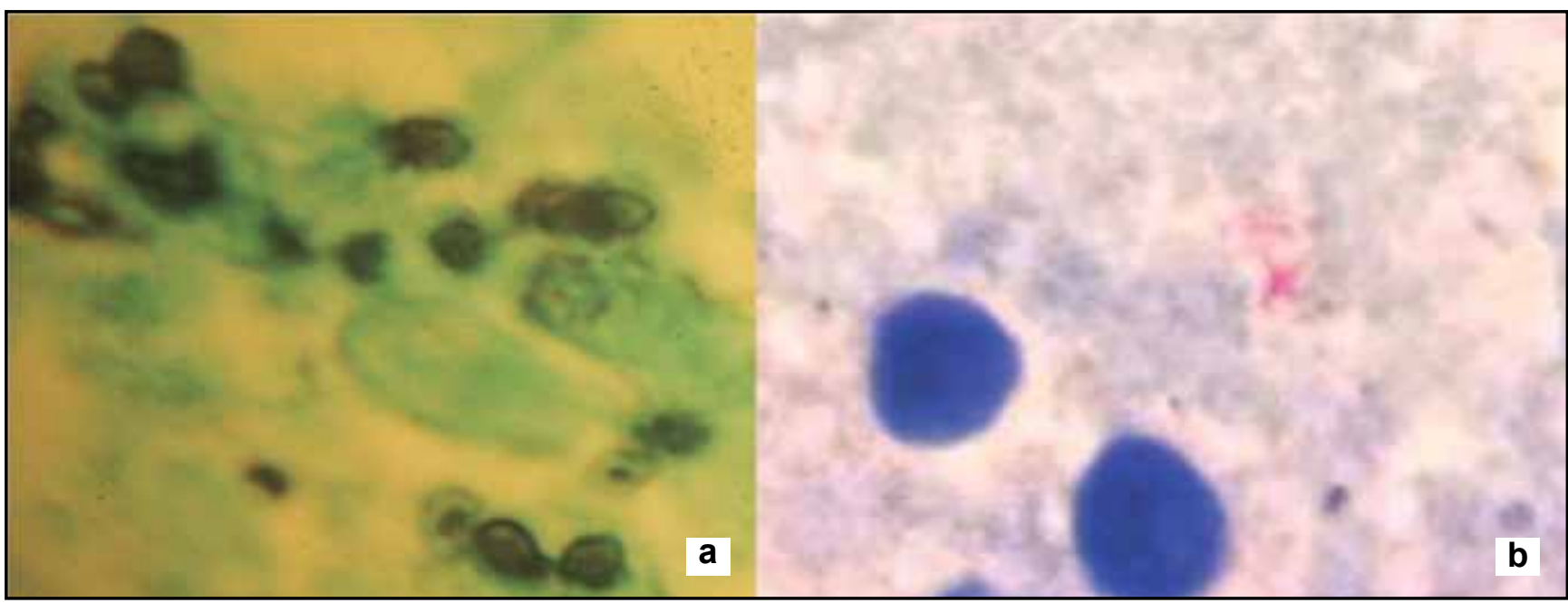

Figura 3. Infección mixta en ovario. a) Coloración de Grocott - levaduras de 2 a 4 micras de diámetro, compatibles con Histoplasma capsulatum. b) Coloración de Ziehl Neelsen - presencia de BAAR.

10.5, proteína $C$ reactiva 4.7. Marcadores tumorales, ELISA para VIH negativo, VDRL no reactivo. Laparoscopia abdominal demostró ascitis; se tomó muestra de peritoneo, ovario, apéndice y epiplón, reportando hallazgos de anatomía patológica compatibles con inflamación crónica, granulomas no necrotizantes, células epitelioides, células gigantes e hiperplasia mesotelial, sin evidencias de malignidad. Por este motivo es referida a nuestra sección con sospecha de enfermedad granulomatosa de origen micótico. Se revisaron las láminas de la muestra del ovario, coloreadas con Grocott, evidenciándose levaduras compatibles con Histoplasma capsulatum. Se inició tratamiento con itraconazol con mejoría parcial. Por este motivo se revisan las láminas coloreadas con Ziehl Nielsen, encontrando bacilos ácido-alcohol resistentes (BAAR) (Figura 3). Se confirma coinfección por Histoplasma capsulatum y Mycobacterium $s p$ y se añade el tratamiento antituberculoso con evolución satisfactoria.

\section{Discusión}

Las infecciones por hongos son cada vez más frecuentes en países endémicos como Venezuela, siendo la histoplasmosis una de las más comunes. En este estudio se describen algunos casos inusuales.

El primer caso muestra una forma de presentación bastante rara de histoplasmosis del sistema nervioso central, el absceso cerebral. En nuestro paciente esta entidad es expresión de una forma diseminada aguda de la enfermedad. Esto ocurre en $10-20 \%$ de los casos. No se conoce aún la razón por la cual el hongo llega a esta localización. Por lo general comienza con un cuadro respiratorio febril, acompañado con tos y poca expectoración, pérdida de peso, anemia y leucopenia $(3,4)$.

La forma más frecuente de infección del sistema nervioso central es la meningitis crónica, la cual se observa en pacientes inmunocomprometidos, por ejemplo en pacientes con
SIDA, en donde no hay evidencia documentada de infección pulmonar primaria hasta en 50\% de los casos. En cambio, en inmunocompetentes se han descrito histoplasmomas y abscesos cerebrales (2). En estos pacientes es frecuente la forma indolente e inespecífica de enfermedad, con síntomas vagos que hacen difícil sospechar el diagnóstico. Esto aunado a la baja sensibilidad de las pruebas micológicas, resulta en un gran porcentaje de casos no diagnosticados que terminan en enfermedad crónica, con elevada morbilidad y mortalidad. Esta última se estima entre 11 y $100 \%$ con un promedio de $25 \%$, aun en pacientes con tratamiento agresivo (4).

El paciente no era VIH positivo ni presentaba otros factores de inmunocompromiso. Posiblemente el origen de la infección se relacionó con el tamaño del inóculo, en vista del antecedente del contacto con una cueva habitada por murciélagos en Boconó, población altamente endémica en nuestro país. Existen reportes de casos que ilustran la dificultad de realizar el diagnóstico de histoplasmosis del sistema nervioso central en pacientes inmunocompetentes, en quienes el único factor de riesgo encontrado fue la exposición previa a un alto inóculo (4).

El diagnóstico precoz requiere un alto grado de sospecha. El hongo debe ser buscado en distintas muestras. En nuestro paciente se realizó mediante el examen directo con Giemsa de la secreción purulenta del absceso, en donde se demostró la presencia del hongo. La inmunodifusión doble resultó positiva y el cultivo fue negativo. Se ha descrito que esta prueba tiene baja sensibilidad, por lo que debe estar acompañada por otros métodos diagnósticos. El tratamiento se realizó con anfotericina B e itraconazol (4-6).

En pacientes inmunocompetentes con antecedentes epidemiológicos positivos, con o sin patología pulmonar, con signos neurológicos inexplicables, como meningitis crónica o lesiones parenquimatosas de etiología desconocida, debe sospecharse esta enfermedad, particularmente si se han descartado otras infecciones como criptococosis y tuberculosis. 
El segundo y tercer caso que se describen, además del hallazgo de infección por $H$. capsulatum en el líquido sinovial de la rodilla y en la biopsia de ovario respectivamente, presentaron evidencia de BAAR y en el líquido de la rodilla hubo crecimiento en cultivo de M. tuberculosis e Histoplasma capsulatum. Estos pacientes tampoco se encontraban en situación de inmunocompromiso ni tenían otras comorbilidades. El único factor predisponente además de la epidemiología, en ambos casos fue la edad mayor de 60 años. Adicionalmente en el caso de la paciente femenina, había el antecedente de limpieza de un ático con abundantes excretas de palomas, era fumadora y presentaba un estado depresivo.

La infección fúngica de huesos y articulaciones, es una entidad poco común, que a menudo se presenta de forma indolente. Candida spp, Paracoccidioides brasiliensis, Coccidioides immitis e Histoplasma capsulatum, entre otros, provocan esta afección. Pueden producir infecciones osteoarticulares en personas previamente sanas (5\% a 10\%) $(7,8)$. La enfermedad se presenta principalmente en niños y adultos jóvenes, aunque puede aparecer a cualquier edad, como es el caso de nuestro paciente, quien tenía 61 años; se acompaña de una forma destructiva de osteomielitis y artritis (NUNCA se describieron los hallazgos imagenológicos). Puede formar parte de un proceso multisistémico o presentarse en forma aislada, como resultado de una diseminación hematógena, por inoculación directa por una fuente exógena o por extensión directa desde un foco adyacente. Aunque cualquier articulación puede estar afectada, se ve con más frecuencia en aquellas que soportan el peso del cuerpo, tales como rodillas, caderas y columna lumbar. El diagnóstico se realiza identificando la presencia del hongo mediante el examen directo, el cultivo y pruebas serológicas. El tratamiento es quirúrgico, acompañado por anfotericina B e itraconazol $(7,8)$.

Las infecciones fúngicas se han convertido en un problema en personas mayores, debido al aumento de la longevidad y de la calidad de vida de este grupo (9). La histoplasmosis en adultos, frecuentemente se comporta como la reactivación de un foco latente, lo cual se encuentra bien documentado en esta enfermedad. Los pacientes ancianos presentan cambios en la inmunidad innata y adaptativa, lo que se denomina inmunosenescencia, por tal motivo, no son capaces de defenderse de microorganismos como H. capsulatum $(9,10)$.

Por otra parte, la tuberculosis articular también constituye una forma de presentación infrecuente, representando 1 - 5\% de los casos de osteomielitis crónica (2).

La tuberculosis osteomuscular se puede manifestar de cinco maneras diferentes, la más común es la enfermedad de Pott (50\%), seguida por artritis periférica (30\%) como en el caso de nuestro paciente, y menos frecuentemente (20\%) dactilitis, tenosinovitis y enfermedad de Poncet (7).

Se ha descrito la coinfección por Histoplasma y M. tuberculosis, en pacientes con SIDA, pero es muy poco frecuente en pacientes inmunocompetentes. La asociación entre estas dos enfermedades no es de extrañar, especialmente en nuestro país, endémico para las dos entidades, en vista de que es conocido que la patogenia de ambas es similar. Por lo tanto, en un paciente mayor de 60 años de edad, con afectación articular, de curso crónico, es importante descartar ambas enfermedades $(2,9,11)$. La evolución del paciente antes mencionado fue muy tórpida, ya que se indicó tratamiento adecuado para la tuberculosis, descuidando el antifúngico.

En los casos que evaluamos en la Sección de Micología Médica del Instituto de Medicina Tropical, Universidad Central de Venezuela, se encontraron otros pacientes con afectación osteoarticular, sin embargo en este caso en particular confluyeron varios aspectos interesantes, tales como la edad mayor de 60 años y la coinfección con tuberculosis. No pudimos encontrar en este paciente antecedentes epidemiológicos ni otros factores de riesgo para la enfermedad. A la luz de nuestros conocimientos es el primer caso descrito en Venezuela de coinfección por M. tuberculosis e $H$. capsulatum de localización articular en un paciente inmunocompetente (11). En la literatura internacional, hallamos un caso, en un diabético (12).

En el tercer caso descrito, se presentó una patología de abdomen y pelvis con hallazgo de Histoplasma sp y bacilos acidorresistentes en el ovario. La histoplasmosis diseminada con este tipo de presentación es extremadamente rara. Han sido reportados casos de enfermedad urogenital en diferentes series de autopsias (13). En éstas la evidencia de la enfermedad se encontraba en las necropsias, aun en pacientes sin signos ni síntomas denominándolas "clínicamente silentes". Esto nos hace suponer que es una entidad más frecuente de lo que parece y que lo más probable es que haya subregistro de casos (13). Isotalo en el año 2000 reportó una paciente con lupus eritematoso sistémico y fístula colorrectal, a quien se realizó una intervención quirúrgica de la misma y como hallazgo casual, se evidenció infección por Histoplasma en el ovario, aunque no había presentado ningún síntoma (14). Por otra parte, la tuberculosis de los ovarios y trompas de Falopio ha sido descrita ampliamente ya que presenta mayor frecuencia de manifestaciones clínicas que la histoplasmosis, por lo cual no sería descabellado pensar que los signos y síntomas que presentó nuestra paciente fueran más debidos a la tuberculosis que a la histoplasmosis, siendo esta última un hallazgo casual (14).

En esta paciente no se pudo dilucidar si se trataba de una reinfección o de una reactivación, en vista de que tenía antecedentes de haber vivido en una granja avícola (de gallinas ponedoras) y uno reciente de limpiar un departamento con abundantes excretas de palomas. Presentaba otros factores de riesgo como son la edad y la depresión, además del hábito tabáquico acentuado.

Hemos descrito tres casos de presentación inusual, en los cuales se llegó al diagnóstico debido a la competencia de profesionales que sospecharon la enfermedad. Además, se observaron presentaciones con afectación del sistema nervioso central, osteoarticular y ginecológica y la asocia- 
ción con tuberculosis. Estas formas son más frecuentes de lo que se piensa, de ahí la importancia de este reporte.

\section{Financiación}

El trabajo no tuvo ninguna fuente de financiación externa a las propias de los autores. Ninguno de los autores declara conflictos de interés.

Ninguno de los autores ha recibido de parte de compañías farmacéuticas o de otros patrocinadores: becas, honorarios por consultorías, honorarios por participación en la revisión de los datos o en el análisis estadístico de la investigación, honorarios por escribir o revisar el manuscrito o apoyo para viajar a reuniones relacionadas con la investigación.

Ninguno de los autores declara actividades financieras relacionadas con compañías farmacéuticas o con otras empresas que, aunque no tengan que ver directamente con el manuscrito enviado, podrían constituir conflictos de interés

\section{Referencias}

1. Kauffman CA. Histoplasmosis: A clinical and laboratory update. Clin Microbiol Rev 2007; 20: 115-132.

2. Agudelo CA., Restrepo CA., Molina DA, TobonAM, Kauffman CA., Murillo C, Restrepo A. Tuberculosis and Histoplasmosis Co-Infection in AIDS Patients. Am. J. Trop. Med. Hyg. 2012; 87 (6): 1094-1098.

3. Chakrabarti A. Epidemiology of central nervous system mycoses. Neurol India. 2007; 55: 191-197.

4. Trofa D, Nosanchuk JD. Histoplasmosis of the Central Nervous System. $J$ Neuroparasitol. 2012; 3: 1-7.
5. Schestatsky P., Chedid MF, Amaral OB, Unis G, Oliveira FM, Severo LC. Isolated central nervous system histoplasmosis in immunocompetent hosts: a series of 11 cases. Scand J Infect Dis. 2006; 38: 43-48.

6. Parihar, V. Tomar, BK, Ojha, Husain N., Gupta RK. Magnetic resonance imaging findings in a patient with isolated histoplasma brain abscess. Arch Neurol. 2011; 68: 534-535.

7. Kemper CA, Deresinski SC. Fungal disease of bone and joint. In: Elias J. Anaissie, MD, Michael R. McGinnis, PhD, and Michael A. Pfaller, MD. Clinical Mycology (Second Edition) chapter 25 2009, Pages 525-545.

8. Kohli R Hadley S. Fungal arthritis and osteomyelitis. Infect Dis Clin North Am. 2005; 19: 831-51.

9. Kauffman CA. Fungal Infections in Older Adults. CID. 2001; 33: 550-55.

10. Ledtke C ,Tomford JW, Jain A, Isada CM, van Duin D. Clinical presentation and management of histoplasmosis in older adults. J Am Geriatr Soc. 2012; 60 (2): 265-70.

11. Mata-Essayag S, Colella M, Rosello A, Hartung C, Landaeta M, Pérez C, Magaldi S, Olaizola C, Calatroni M et al. Histoplasmosis: a study of 158 cases in Venezuela, 2000-2005. Medicine 2008; 87: 193:202.

12. Negroni R, Musella R, Arechavala A, Maiolo E, Rojas H. Problemas clínicos en Micología. Médica: problema no 26 Rev Iberoam Micol 2007; 24: 171-173.

13. Friskel E, Klotz SA., Bartholomew W, Dixon A. Two Unusual Presentations of Urogenital Histoplasmosis and a Review of the Literature. Clin Infect Dis 2000; 31: 189-91.

14. Isotalo PA., McCarthy AE., Eidus L. Ovarian histoplasmosis in systemic lupus erythematosus. Pathology. 2000; 32 (2): 139-141. 